\title{
FUNCIONAMIENTO Y REPERCUSIÓN FAMILIAR EN ADOLESCENTES EMBARAZADAS ATENDIDAS EN EL INSTITUTO NACIONAL MATERNO PERINATAL PERIODO ENERO - MARZO 2013.
}

\author{
Nelly Ojeda Alegría
}

\begin{abstract}
RESUMEN
Objetivo. Caracterizar, desde un enfoque familiar, a las adolescentes embarazadas que se atienden en el Instituto Nacional Materno Perinatal. Materiales y métodos. El universo estudiado comprendió a todas las adolescentes hospitalizadas en el servicio de gineco obstetricia de Adolescencia, durante el periodo de enero a marzo año 2011. Se analizó los informes Sociales, Psicológicos y Médicos de las historias clínica. Fueron estudiadas las variables edad, grado de instrucción, procedencia, estado civil, ocupación, edad de la pareja, ocupación de la pareja, relación de pareja, № de parejas sexuales, № de hijos, ocupación del padre, ocupación de la madre, clasificación de la familia, funcionamiento familiar, $\mathrm{N}^{\circ}$ de hermanos, factores protectores, vinculo afectivo, atención clínica, problemas de salud, problemas sociales, proyecto de vida y repercusión familiar del embarazo en la adolescencia como problema de salud. Resultados. el intervalo de edad predominante en el que ocurrieron los embarazos fue entre los 15 y 18 años, registrándose 312 de los 345 casos $(90.5 \%)$. El nivel de escolaridad se ubicó entre la secundaria incompleta con 195 casos (56.5\%).195 (56.5\%) de las adolescentes eran solteras, desempleadas $223(64.6 \%)$ y 247 (72\%) procedían de Lima. Con respecto a la pareja 206 (59.7\%) son adolescentes, $(49.8 \%)$ inestabilidad de pareja. Los mayores porcentajes correspondieron a las familias constituidas $171(49.5 \%), 125(36.2 \%)$ familias desintegradas, $29(8.4 \%)$ familias incompletas, $20(5.8 \%)$ familias reconstituidas, 190 (55\%) refirieron su dinámica familiar funcional y disfuncional 155 casos $(45 \%)$, y una repercusión moderada $(32,2 \%)$ y favorable $(64,2 \%)$. Conclusiones. Se recomienda estructurar un sistema de acciones educativas $y$, desde un enfoque integral como elemento del ajuste social y psicológico, la participación del Médico en la atención de estas embarazadas adolescentes y de su medio familiar.
\end{abstract}

Palabras clave: Embarazo; Adolescente; Sexualidad; Familia (fuente: DeCS BIREME).

\section{FUNCTIONING AND FAMILY REPERCUSSION IN PREGNANT ADOLESCENTS CARED IN THE NATIONAL MATERNAL PERINATAL INSTITUTE PERIOD OF TIME JANUARY - MARCH 2013.}

\begin{abstract}
Objective. To Characterize, from a family approach, to pregnant adolescents who are cared in the Instituto Nacional Materno Perinatal. Materials and methods. The universe studied embraced all the adolescents hospitalized in the service of obstetrics and gynecology of adolescence, during the period of time from January to March 2011. It analyzed the social reports, psychological and medical histories of the clinic. They were studied the variables age, level of education, origin, marital status, occupation, age of the couple, occupation of the couple, couple's relationship, No. of sexual couples, No. of siblings, occupation of the father, occupation of the mother, classification of the family, family functioning, No. of brothers, protective factors, emotional link, clinical care, health problems, social problems, project of family life and family repercussion of the pregnancy in the adolescent as a health of problem. Results. The age range predominant in that occurred the pregnancies were between 15 and 18 years old, registering 312 of the 345 cases $(90.5 \%)$. The level of education is ranked among the incomplete secondary with 195 cases (56.5\% ).195 (56.5\%) of the adolescents were unmarried, unemployed $223(64.6 \%)$ and $247(72 \%)$ came from Lima. With regard to the couple 206 (59.7\%) are adolescents $(49.8 \%)$ instability of couple. The highest percentages belonged to the constituted families $171(49.5 \%), 125(36.2 \%)$ broken families, $29(8.4 \%)$ incomplete families, $20(5.8 \%)$ reconstituted families, $190(55 \%)$ brought up their family dynamic functional and dysfunctional 155 cases $(45 \%)$, and a moderate repercussion $(32.2 \%)$ and favorable $(64.2 \%)$. Conclusions. It is recommended to structure a system of educational actions and, from a holistic approach as an element of the social and psychological adjustment, the participation of the physician in the care of these pregnant adolescents and their family environment.
\end{abstract}

Key words: Pregnancy; Adolescent; Sexuality; Family(source: MeSH NLM).

\section{INTRODUCCIÓN}

La adolescencia es la etapa de transición durante la cual el niño se transforma en adulto. Durante este período se producen aceleradamente en el ser humano cambios físicos y síquicos muy marcados, entre lo más significativos están la aparición de la primera menstruación o menarquia en la niña. La maduración completa del organismo de la mujer no se limita a su capacidad de fecundación o a las posibilidades de que se produzca un embarazo, por todo esto no es incorrecto plantear que en esta etapa la mujer tiene la madurez suficiente para enfrentar tanto biológica como socialmente una gestación.

La adolescencia es una etapa fundamental de la vida en la cual la y el joven enfrenta cambios físicos, biológicos, sociales, así como de personalidad. Es un período de transición de la niñez a la etapa adulta en la que el

Licenciada en Trabajo Social. Instituto Nacional Materno Perinatal. Lima-Perú.

Recibido: 10-10-13 Aprobado: 3-12-13 
adolescente adquiere valores, aptitudes, actitudes en su forma de pensar, características e identidad propia que le ayudarán a enfrentarse a la vida.

Según la Organización Mundial de la Salud (OMS 2007), la adolescencia se define como: "el período de la vida en el cual el individuo adquiere la capacidad reproductiva, transita los patrones psicológicos de la niñez a la adultez y consolida la independencia socioeconómica; fija sus límites entre los 10 y 20 años" (p.3). Constituye una etapa difícil del ser humano, donde si no existe buena comunicación entre padres e hijos y una excelente educación sexual, muchos de éstos van a truncar sus proyectos de vida.

El embarazo irrumpe en la vida de las adolescentes en momentos en que todavía no alcanzan la madurez física y mental, a veces en circunstancias adversas como son las carencias nutricionales $u$ otras enfermedades, y en un medio familiar generalmente poco receptivo para aceptarlo y protegerlo.

Soto, (2009) sostiene que: El embarazo adolescente es un reto al desarrollo integral de los y las adolescentes. El embarazo adolescente es más frecuente que lo que la sociedad quisiera aceptar. Entre sus posibles causas se encuentra el inicio temprano de la vida sexual de los jóvenes, el uso inadecuado o el no uso de métodos anticonceptivos, así como la falta o insuficiente información sobre sexualidad, el bajo nivel educativo y económico, los bajos niveles de planeación y expectativas que poseen los jóvenes con respecto a su futuro, una pobre imagen y valorización de la mujer, sentimientos de soledad y rechazo, entre otras. (p.3)

El embarazo en la adolescencia, además de constituir un riesgo médico elevado, lleva aparejado dificultades socioeconómicas y emocionales para la madre y su hijo, lo que se conoce como el "Síndrome del fracaso". Es por tanto, la etapa que puede determinar el sentido de la vida y el bienestar futuro (Davis Ivey Ch. 1998).

El problema que se presenta para las embarazadas adolescentes es que están transitando por el difícil camino de transformarse en una mujer adulta, y a la crisis de la adolescencia, desencadenada por los cambios biológicos, psicológicos, sociales e interpersonales, se le suma la crisis del embarazo que es una crisis secundaria provocada por la presencia de una vida en el vientre, que compromete a estas jóvenes con una responsabilidad y madurez para las que no se han desarrollado totalmente (González G.H. 2006).

Jeannevive (2007): Señala a la familia es uno de los microambiente donde permanece el adolescente y por lo tanto es responsable de lograr que su función favorezca un estilo de vida saludable, el que debe promover el bienestar y desarrollo de sus miembros. Como unidad psicosocial, la familia está sujeta a la influencia de factores socioculturales, protectores y de riesgo, cuya interacción la hace vulnerable a caer en situaciones de crisis o en patrones disfuncionales.

En efecto, cuando la familia funciona de forma adecuada es uno de los focos de prevención primordial y primaria de embarazo en la adolescente. La familia bien constituida, por lo general, protege a las chicas de caer en situaciones de riesgo de quedar embarazada, puesto que están pendiente de los amigos con quien andas, la hora que sale del colegio, es decir, controlan sus entradas y salidas del hogar y del lugar donde se dirigen.

Asimismo, Stern (2007), plantea la existencia de familias disfuncionales, donde las relaciones entre padres o tutores son inadecuadas, basadas en riñas, agresiones y faltas de respetos, esta situación genera carencia afectiva, desapego, en consecuencia, impulsa a la adolescente a la práctica de relaciones sexuales, en busca de ese amor que no tiene en el hogar.

En el Instituto Nacional Materno Perinatal, se efectúa la atención multidisciplinaria a las adolescentes embarazadas, donde todas son valoradas por diferentes especialidades, dentro de ellas, la atención social. Esta atención tiene como objetivo principal identificar aquellas adolescentes embarazadas de riesgo clínico social, con el propósito de mejorar el bienestar social y psicológico de la adolescente embarazada.

Por todo lo expuesto y considerando que el embarazo en la adolescencia es un problema de salud con repercusión biopsicosocial, es que nos propusimos realizar este estudio con el objetivo de caracterizar desde la percepción familiar, a las adolescentes embarazadas, hospitalizadas en el servicio de Adolescencia del INMP, y precisar el grado de satisfacción familiar percibido por las embarazadas adolescentes, describir las relaciones familiares presentes en la muestra estudiada y valorar la percepción del funcionamiento familiar en las adolescentes en estudio.

\section{MATERIALES Y METODOS}

Se ha realizado un estudio descriptivo, de tipo retrospectivo en el que, desde la percepción familiar, se caracterizó a una población de 345 adolescente embarazada, hospitalizadas en el servicio de gineco obstetricia de adolescencia, que fueron atendidas en el Instituto Nacional Materno Perinatal, en el período de Enero a marzo del 2011, cuyo rango de edad osciló entre los 10-18 años. Se tomaron como:

\section{Criterios de inclusión:}

Rango de edad: 10-18 años.

Pertenecer al Área de hospitalización del servicio de gineco obstetricia de adolescencia del INMP. 


\section{Criterios de exclusión:}

- Adolescentes de consulta externa.

- Familiar de la adolescente embarazada, que presente alguna limitación mental.

Al seleccionar la muestra se tomó la edad como variable epidemiológica y como variables psicosocial la clasificación familiar, funcionamiento familiar y repercusión familiar.

CLASIFICACIÓN DE LA FAMILIA: Se refirió a los distintos tipos de familia según tamaño y ontogénesis.

\section{Tamaño:}

- Grande: (7 o más miembros)

- Mediana: (de 4 a 6 miembros)

- Pequeña: (de 1 a 3 miembros)

Ontogénesis: Se tuvo en cuenta la presencia de uno o ambos padres, clasificándola en:

- Familia constituida.

- Familia desintegrada.

- Familia incompleta.

- Familia reconstituida.

FUNCIONAMIENTO FAMILIAR: Expresó la dinámica de las relaciones entre los miembros de la familia, la cual se produce en forma sistémica y es relativamente estable. Condiciona el ajuste de la familia al medio social y el bienestar subjetivo de sus integrantes.

- Familia funcional.

- Familia disfuncional.

REPERCUSIÓN FAMILIAR: Expresó el impacto que tiene el evento vital que atraviesa la familia con el (embarazo de la adolescente), exigiéndole reajustes, cambios o modificaciones en su dinámica interna y modo de vida, que pueden favorecer la salud o dificultarla.

- Repercusión favorable: Cuando la familia evalúa - considera que los cambios y modificaciones realizadas, o el hecho de no haber ocurrido cambios son favorables para el sistema familiar.

- Repercusión desfavorable: Cuando la familia evalúa - considera que los cambios y modificaciones realizadas, o el hecho de no haber ocurrido cambios, son desfavorables para el sistema familiar.

\section{RESULTADOS}

La maternidad precoz es un fenómeno social por su crecimiento y efectos que producen. El $46 \%$ de las entrevistadas tienen entre 17 y 18 años, el $44.5 \%$ son menores de 15 a 16 años, el $9 \%$ tienen entre 13 y 14 años y el $0.5 \%$ tiene entre 10 a12 años, siendo la maternidad precoz un problema social que demanda atención e intervención profesional del equipo multidisciplinario.

La pobreza asociada a los bajos niveles de educación es uno de los factores subyacentes del embarazo precoz, ya que la mayoría de madres abandonan la educación, porque el sistema educativo no lo permite o porque ellas se sienten diferentes a sus pares y deciden no continuar sus estudios, resultados de la investigación demuestran que el $56.5 \%$ de adolescentes tienen secundaria incompleta, el 33.3\% secundaria completa, y el $9.3 \%$ tienen primaria completa o incompleta, siguiéndole el técnico medio con $0.9 \%$.

El hecho de tener una relación informal de pareja parece ser determinante del embarazo de la adolescente, el $56.5 \%$ de las entrevistadas manifestaron ser madres solteras, ya que el embarazo se produce en su mayoría por acto involuntario, donde las adolescentes por curiosidad o sensibilidad amorosa experimentan su primera relación sexual, sin medir consecuencias, y el $43 \%$ refirió convivencia donde las adolescente madres simplemente viven juntos en casa de los padres dependiendo económicamente.

El $64.6 \%$ de las adolescentes se encuentran desempleadas, dedicadas al hogar, dicha situación está condicionada por su estado de embarazo, que les obliga a interrumpir cualquiera actividad renumerada y depender del apoyo de la pareja, padres y otras personas, el $26 \%$ tienen perspectivas de seguir estudiando y el $9.2 \%$ trabajan desempeñando oficios de baja productividad, como empleadas del hogar, vendedor ambulante, (sin beneficios de ESSALUD).

De la población atendida el $60.3 \%$ refirió tener un hijo, el $3.5 \%$ ya habían tenido dos a tres hijos, y el $36.2 \%$ en espera del nacimiento. Los bebes de madres Adolescentes tiene una alta tasa de mortalidad al igual que pueden experimentar mal formaciones congénitas, problemas de desarrollo, retraso mental, ceguera, o parálisis cerebral. El $80 \%$ de adolescentes declararon tener una sola pareja sexual, el $20 \%$ menciona tener una segunda pareja sexual, habiendo sido abandonada por sus anteriores parejas (Tabla 1).

Las madres adolescentes con pareja, se puede inferir que presentan cierta ventaja en cuanto a nivel de proyecto de vida, esta situación aun cuando se presenta como ventaja no es igual para todos ya que el $59.7 \%$ de parejas son adolescentes, que comparten vivienda y reciben apoyo diverso de la familia de origen, el $28.7 \%$ sus edades fluctúan entre 21 y 25 años, el $8.1 \%$ las edades fluctúan 26 y 30 años, y el $1.8 \%$ son mayores de 31 años y el $1.7 \%$ manifiesta desconocer.

El $30 \%$ de las parejas de las adolescentes, manifestaron realizar trabajos eventuales, como cobrador, ayudante de gasfitería, pintor, vigilante, la mayoría de ellos sin acceso a ESSALUD, el $16.2 \%$ efectúa trabajos independientes 
(zapatero, carpintero, chofer, albañil, electricista, mecánicos), con capacitación formal o por experiencia adquirida, en la que se desempeñan permanentemente.

El $20 \%$ declararon laborar como obreros en el sector privado, en condiciones laborales inadecuadas, sin acceso a los beneficios sociales de los trabajadores estables. El $9.8 \%$ se dedican al trabajo informal y/o venta ambulatoria, el $15.4 \%$ son estudiantes, el $5.2 \%$ son desempleados y el $3.4 \%$ no conoce la actividad laboral.

La relación de pareja de las adolescentes están llenas de diversos matices, el 60 \% refirió una relación no estable, ya que las adolescente en su mayoría tienen como parejas a personas mayores que ellas, lo que implica muchas veces condición de compañeros que se comporten con una madurez muy por encima a la acostumbrada por la adolescente. $Y$ el $40 \%$ de las adolescentes manifestaron una relación estable (Tabla 2).

Según la categoría de ocupación destaca que el $31.9 \%$ de los padres de las adolescentes son trabajadores independientes, le sigue en importancia el trabajo eventual con un $21.5 \%$, el $19.7 \%$ están como obreros, el $8.1 \%$ se dedican al comercio informal, el otro $8.1 \%$ desconoce la actividad laboral del padre por reconocer paradero, el $7.8 \%$ son fallecidos, y el $2.9 \%$ son trabajadores estables En el caso de las madres de las adolescentes, en un $41.5 \%$ manifestaron dedicarse a los quehaceres del hogar, el $22 \%$ se dedica a la venta ambulatoria, el $11 \%$ son empleadas del hogar, el $9.6 \%$ se dedican al trabajo independiente, el $6.1 \%$ tienen trabajo estable, el $4.3 \%$ son contratadas, el $2.9 \%$ son fallecidas y el $2.6 \%$ desconoce la actividad laboral.

El tipo de vivienda en el que habita la madre adolescente es de propiedad familiar en un $79.1 \%$, es decir viven en

Tabla 2. Características de la pareja de la adolescente. Servicio de Adolescencia. INMP 2011

\begin{tabular}{lcc}
\hline CARACTERISTICA & $\mathbf{N}^{\mathbf{0}}$ & $\%$ \\
\hline Edad de la pareja & & \\
$15-20$ & 206 & 59.7 \\
21 - 25 & 99 & 28.7 \\
26 - 30 & 28 & 8.1 \\
31 - 35 & 03 & 0.9 \\
36 A + & 03 & 0.9 \\
Desconoce & 06 & 1.7 \\
Ocupación & & \\
Estudiante & 53 & 15.4 \\
Trabajador Independiente & 56 & 16.2 \\
Trabajador Eventual & 103 & 30 \\
Obrero & 69 & 20 \\
Vendedor ambulante & 34 & 9.8 \\
Sin empleo & 18 & 5.2 \\
Desconoce & 12 & 3.4 \\
Relación de pareja & & \\
Estable & 138 & 40 \\
No estable & 207 & 60 \\
\hline
\end{tabular}

condición de alojadas, en casa multifamiliares y hacinadas, el $12.5 \%$ tienen accesibilidad de vivir en casas alquiladas, el $7.5 \%$ declara vivienda en calidad de guardianía y el $0.9 \%$ viven en hogares tutelares.

Las relaciones familiares influyen considerablemente en el comportamiento sexual de la adolescente. Siendo más probable que las adolescentes que pertenecen a familias desestructuradas, ya sea por separación y/o divorcio de los padres busquen compensación emocional en la actividad sexual y/o embarazo para suplir la falta de afecto dentro del entorno familiar. Como vemos en la inv. Efectuada el $49.6 \%$ de las adolescentes proceden de hogares constituidas, el $36.2 \%$ proceden de hogares desintegrados el $8.4 \%$ de familias incompletas, y el $5.8 \%$ proceden de familias reconstituidas.

Respecto al funcionamiento familiar en las madres adolescentes, el $55 \%$ refirió tener una familia integrada funcional, sin embargo todas buscan una especie de refugio en una pareja que suplirá en amor que no han recibido de sus padres, y el $45 \%$ de adolescentes tenían

Tabla 3. Entorno familiar de la adolescente. Servicio de Adolescencia. INMP 2011

\begin{tabular}{|c|c|c|}
\hline CARACTERÍSTICA & $\mathbf{N}^{\circ}$ & $\%$ \\
\hline \multicolumn{3}{|l|}{ Ocupación del padre } \\
\hline Trabajador Independiente & 110 & 31.9 \\
\hline Trabajador Eventual & 74 & 21.5 \\
\hline Obrero & 68 & 19.7 \\
\hline Comercio informal & 28 & 8.1 \\
\hline Empleado & 10 & 2.9 \\
\hline Desconoce & 28 & 8.1 \\
\hline Fallecido & 27 & 7.8 \\
\hline \multicolumn{3}{|l|}{ Ocupación de la madre } \\
\hline Su casa & 143 & 41.5 \\
\hline Vendedor Ambulante & 76 & 22 \\
\hline Empleada del hogar & 38 & 11 \\
\hline Independiente & 33 & 9.6 \\
\hline Empleada & 21 & 6.1 \\
\hline Contratada & 15 & 4.3 \\
\hline Fallecida & 10 & 2.9 \\
\hline Desconoce & 09 & 2.6 \\
\hline \multicolumn{3}{|l|}{ Vivienda } \\
\hline Alojada & 273 & 79.1 \\
\hline Alquiler & 43 & 12.5 \\
\hline Guardianía & 26 & 7.5 \\
\hline Hogar tutelar & 3 & 0.9 \\
\hline \multicolumn{3}{|l|}{ Tipo de familia } \\
\hline Familia constituida & 171 & 49.6 \\
\hline Familia desintegrada & 125 & 36.2 \\
\hline Familia incompleta & 29 & 8.4 \\
\hline Familia reconstituida & 20 & 5.8 \\
\hline \multicolumn{3}{|l|}{ Dinámica familiar } \\
\hline Familia funcional & 190 & 55 \\
\hline Familia disfuncional & 155 & 45 \\
\hline \multicolumn{3}{|l|}{ Tamaño familiar } \\
\hline Pequeña & 98 & 28.4 \\
\hline Mediana & 208 & 60.3 \\
\hline Grande & 39 & 11.3 \\
\hline
\end{tabular}


hogares disfuncionales, ya que muchas de ellas tenían a sus padres separados, vivían con algún familiar, o con los abuelos. Incluso la relación entre las adolescentes y los padres no siempre era buena.

Asimismo vemos que el $71.6 \%$ de hogares son de familias extendidas y numerosas, donde el número de integrantes es de 4 a más de 10 miembros, incrementándose el embarazo precoz, ya que estos hogares se encuentran inmersos en situación de pobreza, y a los bajos niveles educativos de los padres y la adolescente, lo que hace que padres e hijos estén desinformados sobre aspectos de sexualidad y que posiblemente no se produzca una adecuada comunicación entre ellos.

Las madres adolescentes, el $47.6 \%$ consideran que sus proyectos de vida se lograran a través de los estudios, estos les van a permitir en el futuro lograr mejorar sus condiciones de vida y la de sus hijos, el $34.2 \%$ manifestaron cuidar a su recién nacido, el $9 \%$ afirmo trabajando, el $7.8 \%$ dedicarse a los oficios del hogar y el $1.4 \%$ no sabe qué hacer.

Por otra parte, en relación al vínculo afectivo con los miembros de la familia, el $48.4 \%$ consideró el apoyo favorable por parte de la madre cuando quedaron embarazadas, el $18 \%$ de ambos padres, el $12.7 \%$ de la pareja, el $6.3 \%$ del padre, el $6.3 \%$ de otros, y el 0.3 $\%$ fueron abandonadas, teniendo apoyo en las casas hogares.

En el estudio de 345 adolescentes, en casi todas las familias hubo cierta repercusión ante el evento del embarazo de la adolescente, reportándose el $29.6 \%$ con repercusión leve, el $23.8 \%$ repercusión moderada, $13.6 \%$ repercusión elevada, y $7.5 \%$ presento repercusión severa.

Dentro de los problemas sociales que presenta la madre adolescente vemos que el $6.4 \%$ refieren abandono social, el $2 \%$ fármaco dependencia, $5.2 \%$ sufrió violencia sexual, el $55.1 \%$ manifestó soporte familiar inadecuado, el $13.3 \%$ refirió violencia familiar, el $16.5 \%$ refirió tener problemas de reconocimiento y pensión alimenticia y el $0.9 \%$ vivir en hogares tutelares por abandono moral y material de los padres(Tabla 4).

Tabla 4. Problemas sociales que presenta la adolescente servicio de adolescencia. INMP 2011

\begin{tabular}{lcc}
\hline PROBLEMAS SOCIALES & $\mathbf{N}^{\mathbf{0}}$ & $\%$ \\
\hline Abandono social & 22 & 6.4 \\
\hline Farmacodependencia & 07 & 2 \\
Prostitución & 02 & 0.6 \\
Violencia sexual & 18 & 5.2 \\
Violencia familiar & 46 & 13.3 \\
Soporte familiar. Inadecuado & 190 & 55.1 \\
Reconocimiento y pensión alimenticia & 57 & 16.5 \\
Hogares tutelares & 03 & 0.9 \\
TOTAL & 345 & 100 \\
\hline
\end{tabular}

\section{DISCUSIÓN}

El embarazo a cualquier edad constituye un hecho biopsicosocial muy importante, pero en la adolescencia conlleva a una serie de situaciones que puede atentar contra la salud de la madre y la de su futuro hijo; nuestros resultados coinciden con autores que describen en sus investigaciones, que en los últimos años los embarazos entre las adolescentes de 15-19 años han aumentado en un 25\%. Según los resultados obtenidos en este estudio se encontró que el embarazo en la adolescencia es más frecuente en las edades de 13 a 16 años, con un $53.5 \%$, siendo esta edad en factor desfavorable, ya que se conoce que, mientras mas cercano a la menarquia es el embarazo, al no tener las condiciones físicas y psicológicas requeridas, existe mayor riesgo de complicaciones maternas y neonatales.

Ibarra (2003) señala que la repercusión de la maternidad en la esfera escolar de la adolescente, será vivenciada de manera diferente en función del lugar que ocupe la superación en la esfera afectiva motivacional de la adolescente. El comportamiento del nivel de escolaridad de la adolescente embarazada predominó el nivel de secundaria incompleta con un $56.5 \%$. Lo que coinciden con estudios que explican que la mayoría de las adolescentes interrumpieron los estudios por la gestación y para atender a su bebe, y se evidencia la disminución de las expectativas de prolongar su educación y, por lo tanto, la probabilidad de mejorar las condiciones económicas.

En el nivel ocupacional vimos que el $64.6 \%$ de las adolescentes embarazadas se encuentran desempleadas, dedicadas al hogar. A pesar de que el estudio es la labor fundamental en esta etapa de la vida, la gran mayoría de los jóvenes dejan sus estudios de forma precoz y se quedan en la casa, por lo tanto no reúnen los requisitos necesarios para obtener algunos empleos, además, en el país ha existido un alto índice de desempleo ha ido y pensamos que continúe disminuyendo poco a poco con el tiempo. Estos resultados coinciden con estudios de otros autores, donde predomina el grupo de embarazadas adolescentes amas de casa, aunque con cifras mayores.

En relación al estado civil, prevalecen las embarazadas solteras, ocupando más de un $56.5 \%$, y $43 \%$ convivientes, lo que se explica por el mismo hecho de que dejan de estudiar a edades tempranas y al no tener solvencia económica se unen a un hombre se dedican a los hijos. En este caso los datos nuestros coinciden con otros trabajos realizados en Venezuela se ha visto que la mujer adolescente embarazada es madre soltera en el $70 \%$ de los casos, considerando además que el matrimonio contribuye poco a la estabilidad emocional y social de la adolescente, sobre todo cuando se efectúa bajo presión familiar, repercutiendo luego en un mayor porcentaje de divorcios para las mujeres que contrajeron nupcias antes de los 20 años.

La separación de los padres y la crianza de las adolescentes por la madre solamente, favorecen la presencia del 
embarazo en sus hijas con mayor frecuencia. Podemos corroborarlo en algunas investigaciones que concluyen, al señalar, que las familias donde la autoridad moral es débil, pobremente definida, de padres sin casarse, padres múltiples o ausentes, donde las jóvenes han sido criadas por diferentes personas, en diferentes momentos de su vida presentan con más frecuencia embarazos en la adolescencia. Puede explicarse también por la importancia de la figura masculina en la educación de la adolescente, ya sea por el rigor que impone en ocasiones el padre a la hija en la sexualidad, o por la necesidad afectiva de esta, que necesita la figura masculina, y en estos casos tiende a buscarla en otro hombre.

Relacionado con esto, en el estudio se encontró el $45 \%$ son familias disfuncionales. Esto nos demuestra destacar que en todas las familias monoparental que se estudiaron era la figura materna la que vivía con las adolescentes embarazadas, estando ausente la figura paterna. Estos resultados se corresponden con otros alcanzados en donde el promedio de miembros en la familia se encuentra entre 4,6 y 7,9 miembros, por lo que se caracteriza de ser una familia de tamaño mediano. La ausencia de la figura paterna conduce a un incremento significativo de la jefatura femenina en el hogar en los últimos años, situación que genera que la mujer se sienta sobreexigida, sobrecargada en el plano emocional, económico y funcional, debido a la concentración de roles y tareas, teniendo que buscar trabajos que remunerados, lo que, muchas veces, a consecuencia de los horarios irregulares, le da un peso importante a las abuelas en la educación de los hijos y esto conlleva a diferencias generacionales en el medio familia.

La familia, eslabón más importante de la cadena humana, tiene una función decisiva en la educación de los hijos, incluyendo la sexual, de modo que la separación entre padres e hijos trae como resultado numerosos trastornos. La familia con un solo padre suele traer consigo problemas tanto en lo que compete a los hijos, como en la estabilidad emocional del progenitor, asumiendo la responsabilidad en la toma de decisiones y enfrentamiento a las crisis normativas y para normativas del ciclo vital de la familia, lo que incide en la funcionalidad familiar.

En algunas familias pudo observarse que, a pesar de que el embarazo en la hija adolescente implicó grandes cambios al sistema familiar, éstas le atribuyeron una connotación favorable al evento, brindando el apoyo necesario para el curso de la gestación. Sin embargo, otras familias percibieron de manera negativa o desfavorable este acontecimiento, lo cual podría ser amenazante y poner en peligro el bienestar y la salud del sistema familiar.

Teniendo en cuenta los diferentes niveles de impacto y el significado favorable o desfavorable que la familia le asignó al evento, consideramos que el embarazo en la adolescencia puede reflejar diferentes niveles de afectación o repercusión a la familia, también puede ser un riesgo para la salud de la familia, y, en otras familias, puede no presentarse afectación por no representar un problema de salud al sistema familiar, sino por el contrario, lograr una evolución a partir de los cambios o transformaciones necesarios para el desarrollo de la misma.

En la esfera socioeconómica el principal aspecto que se afectó negativamente, fue la dificultad en recursos económicos de la familia para afrontar el evento, por ser la mayoría de las familias núcleos monoparentales; las madres de las adolescentes se sintieron sobrecargadas en cuanto a ciertas demandas que requieren de recursos económicos, como alimentación de la embarazada y satisfacción de necesidades. por lo que, en esta área, se produjo un impacto importante en las familias con cierto sentido negativo, lo cual es fácilmente explicable por el reajuste en el estilo de vida de la familia ante la llegada de un nuevo miembro, pudiéndose generar una crisis por incremento y desorganización. En cuanto a estos resultados, coincidimos con otros estudios realizados en el extranjero (Florenzano, U. 1995; Florenzano R, Pino P, Kaplan M., 1994

Cuando analizamos las familias que presentaron una repercusión elevada en el funcionamiento familiar, es importante recordar que de estas familias estudiadas un alto porcentaje presentó dificultades en su funcionamiento, destacándose 102 familias presentaron la repercusión familiar leve, 10 disfuncionales y tres severamente disfuncionales, por lo que es de esperar que refirieran afectación en esta variable. La presencia de conflictos generacional propia de la etapa de adolescencia, las dificultades en la comunicación y en la esfera afectiva de la familia, pudieron ser principales causantes del impacto negativo en el funcionamiento familiar, afectándose la armonía y cohesión del grupo familiar.

De forma general, podemos decir, en cuanto a la repercusión familiar sobre este evento, que para la mayoría de las familias estudiadas ha sido un momento importante y con consecuencias positivas, es decir, favorable al desarrollo de la familia como sistema. Esto reafirma una vez más la importancia de la evaluación en la familia sobre la repercusión y el impacto de los eventos y su relación con los estilos de afrontamiento de la familia, como primer sistema de apoyo del individuo.

De los resultados de este estudio podemos considerar que la gestante adolescente es una paciente especial, desde el punto de vista biopsicosocial, y que, por tanto, requiere de atención especializada por un equipo multidisciplinario, haciendo particular énfasis en la prevención de las afectaciones del embarazo precoz en la esfera personal y familiar, así como el manejo adecuado de los problemas que se presenten durante su seguimiento.

Con este trabajo señalamos una vez más que el rol que desempeña la familia ante el embarazo precoz, resulta decisivo para optimizar la salud, el bienestar y la calidad 
de vida de la gestante adolescente y del recién nacido, tratándose de un complejo proceso en que no sólo es importante tomar en consideración los riesgos biológicos sino también importantes factores psicosociales que contribuyen en el desarrollo de la nueva sociedad.

\section{RECOMENDACIONES}

Se recomienda estructurar un sistema de acciones educativas y desde un enfoque integral como elemento del ajuste social y psicológico, la participación del Médico en la atención de estas embarazadas adolescentes y de su medio familiar.

\section{REFERENCIAS BIBLIOGRÁFÍCAS}

1. Issler JR. Embarazo en la adolescencia. Revista de Posgrado de la Cátedra Vla Medicina, 2001; 107: 11-23.

2. Molina R, Sandoval J, Luengo X. Obstetricia y Perinatología.

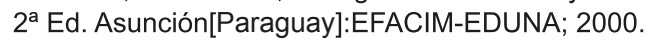

3. Coll A. Embarazo en la adolescencia. Clínicas Perinatológicas Argentinas, 1997; 4: 5-11.
4. Molina R, Abreu M, Sandoval J. Adolescencia Femenina. En: Jadresic A., Ojeda C, y Perez G. eds., Psiconeuroendocrinología. España: Editorial Mediterraneo, 1999.

5. Kinberg M, Coleman J, Hendry L. Psicología de La Adolescencia. 4ta Ed. Madrid: Ediciones Morata; 2003.

6. García Sánchez M, Hernández Hernández ML, Manjon Sánchez A. Embarazo y adolescencia. Rev. sobre Salud Sexual y Reproductiva, 2000; 2: 10-12.

7. Toro Merlo J, Uzcátegui Uzcátegui O. Embarazo en la adolescente. En: Ginecología, Fertilidad y Salud Reproductiva. Caracas: Edit. Ateproca; 2001.

8. Molina R, Pérez A, Donoso E. Adolescencia y embarazo. $2^{a}$ Ed. Santiago de Chile: Técnicas Mediterráneo; 1992.

9. Marchiano MD. Embarazo y adolescencia. Philadelphia: University of Pennsylvania Medical Center; 2002.

10. Coll A. El embarazo en las edades extremas de la vida reproductiva. Argentina: PROAGO; 1998.

11. Issler J. Embarazo en la adolescencia. Rev de postgrado cátedra 6 de Med 2001; 107(10):11-23.

12. Monterroso A, Bello A. Atención obstétrica en adolescentes menores de 15 años. Rev Col Obst Ginecol 1996; 47(1): 15-22.

13. Sánchez P, Mendieta N. Embarazo en la adolescencia: experiencia de la A.S.C.P. Rev Col Obst Ginecol 1994; 45(3): 208-212. 